\title{
Malnutrition as a Major Burden for Under-Five Infants Associated with Malaria in Developing Countries: A Review Study
}

\author{
Authors \\ Dr Md. Shafiur Rahman ${ }^{1}$, Dr M M Aktaruzzaman², Dr Ashekur Rahman Mullick³, \\ Prof Dr Manzurul Haque Khan, $\mathrm{PhD}^{4}$, Dr Irin Hossain ${ }^{5^{*}}$ \\ ${ }^{1,5}$ National Institute of Preventive and Social Medicine, Dhaka, Bangladesh \\ ${ }^{2}$ Dhaka Medical College and Hospital, Dhaka, Bangladesh \\ ${ }^{3}$ Delta Medical College and Hospital, Dhaka, Bangladesh \\ ${ }^{4}$ Directorate General of Health Services, Dhaka, Bangladesh
}

*Corresponding Author

Dr Irin Hossain

\begin{abstract}
This review study aims at discussing about themalnutrition status of under-five children associated with malaria in developing countries. Malaria is a preventable and treatable mosquito-borne disease, whose main victims are children under five years of age. This human parasitic disease remains a major cause of morbidity and mortality worldwide, particularly in developing countries. It affects children below the age of 5 years and pregnant women more severely. The World Malaria Report 2012 summarizes data received from 104 malaria-endemic countries and territories for 2011. Ninety-nine of these countries had on-going malaria transmission. As per the World Malaria Report released on 4 December 2019, in 2018 WHO South-East Asia Region had an estimated 8 million cases and 11600 malaria deaths $-69 \%$ and $70 \%$ less as compared with 2010. This is the largest decline among all six WHO Regions.the relation between the different components of malnutrition: wasting, stunting, and micronutrient deficiencies need further study so that their relation with malaria in under-five children is better understood. Such information could help in designing intervention programmes that promote the survival of children.
\end{abstract}

Keywords-Malnutrition, Under-Five Infants, Malaria, Developing Countries.

\section{Introduction}

Malaria is a preventable and treatable mosquitoborne disease, whose main victims are children under five years of age. This human parasitic disease remains a major cause of morbidity and mortality worldwide, particularly in developing countries. It affects children below the age of 5 years and pregnant women more severely. The World Malaria Report 2012 summarizes data received from 104 malaria-endemic countries and territories for 2011. Ninety-nine of these countries had on-going malaria transmission. In South East Asia, the second most affected region in the world, India has the highest malaria burden (with an estimated 24 million cases per year), followed by Indonesia and Myanmar, ${ }^{1,2}$. As per the World Malaria Report released on 4 December 2019, in 2018 WHO South-East Asia Region had an estimated 8 million cases and 11600 malaria deaths - $69 \%$ and $70 \%$ less as compared with 2010. This 
is the largest decline among all six WHO Regions. Two countries in the Region - Maldives and Sri Lanka - have been certified malaria free, and two more, Timor-Leste and Bhutan, are close to elimination target ${ }^{1}$.

Hunger and malnutrition also remain among the most devastating problems facing the majority of the world's poor and needy, and continue to dominate the health of the world's poorest nations. The United Nations Food and Agriculture Organization estimates that nearly 870 million people, or one in eight people globally, suffered from chronic undernourishment between 2010 and 2012. Nearly $30 \%$ of the population of the developing world, including infants, children, adolescents, adults and older persons, are currently suffering from one or more of the multiple forms of malnutrition. Based on the 2013 United Nations Children's Fund report, 38\% of children below the age of 5 years suffer from chronic malnutrition or stunting in sub-Saharan Africa, with malaria and undernutrition being the two major causes of childhood mortality ${ }^{2}$.

Approximately 12 million children younger than 5 years of age die every year; most of these children live in developing countries. More than $50 \%$ of these deaths are attributed to diarrhea, acute respiratory illness, malaria, or measles, conditions that are either preventable or treatable with low-cost interventions ${ }^{3}$.

In developing countries, malnutrition represents an additional burden and often co-exists with malaria ${ }^{4}$. While under nutrition increases the general susceptibility of an individual to viral, bacterial and parasitic infections ${ }^{5,6}$, infections negatively impact on the nutritional status, resulting in a vicious cycle of under nutrition and infection ${ }^{7,8}$. Synergism between malnutrition and infection is responsible for much of the excess mortality among individuals in less developed regions.

\section{Materials and Methods}

Basically we searched for literatures published either in English or with an English abstract in any foreign-language publication by the help of following groups of keywords: mortality, death; malnutrition, protein energy-malnutrition, anthropometry; malaria, plasmodium.

\section{Selection Criteria \\ Inclusion Criteria}

Two types of studies were included in the table of this review article:

- The first were original research analysis of cohort, cross-sectional and case-control studies that contained data on malaria induced mortality in children stratified by nutritional status assessed using anthropometric indicators or a history of clinical signs of malnutrition (that is, kwashiorkor, marasmus, edema, or recent severe weight loss).

- The second were critical review articles that contained quantitative data and reports of original research results.

\section{Exclusion Criteria}

The following types of studies were not included for this review article:

- Reports of case series;

- Studies in which causes of death was other than malaria in under-five children;

- Studies that used the term malnutrition without any further descriptive information or reference to a source that would provide such information;

\section{Indicators}

In explaining malnutrition, the majority of studies used indicators of anthropometric status like,

- Weight for age.

- Weight for height.

- Mid-upper arm circumference.

Weight for age was the most frequently reported indicator. Some of the hospital-based studies reported a clinical assessment of kwashiorkor, marasmus, or edema.

The case definition for malaria also varied among the studies. Some defined malaria on the basis of a simple history of,

- Fever 
- Malaria cases with clinical signs like history of fever or fever and chills

- Parasitaemia confirmed by microscopic examination of slides.

\section{Data extraction}

One reviewer extracted the following data from relevant studies: year, country, population, design, number of subjects, age range, malaria endemicity, Plasmodium species, intervention, duration and timing, malaria episode definition, exposure, stunting reference standard, outcome, statistical model, covariates, results, effect estimate, and limitations.

\section{Results}

The data from hospital-based studies conducted in Africa suggest that anthropometric status at admission is associated with subsequent death from malaria $^{9-14}$. The largest study involved a cohort of over 8000 cases of malaria and over 700 deaths in the Gambia. Compared with children who had weight for age Z-scores $>-2$, case fatality rates were two fold higher among children with scores of $<-3$ to $>-4$ and three fold higher among those with scores $<-49$. Likewise, data from the Republic of Chad $^{14}$, Madagascar ${ }^{12,13}$, Nigeria ${ }^{11}$, and Senegal ${ }^{10}$ indicate that malnourished children admitted to hospital for severe malaria proceeded less well than adequately nourished cases. No community-based studies were identified.

A cross-sectional study involving 454 children $\leq 5$ years was carried out from February to May 2013 in Muea community found that Clinical malaria parasitaemia was significantly higher $(\mathrm{P}=0.01)$ in children who were stunted $(16.9 \%)$ and underweight $(21.6 \%)$ than their normal counterparts $\left(7.5 \% ; 8.2 \%\right.$ respectively) ${ }^{15}$ (Table 01).

A hospital-based study was conducted from January to December 2007 on 225 consecutive pediatric admissions of malaria cases and forty-five children who had come for vaccination or counseling (control) in four hospitals in Douala found that mild undernutrition was more prevalent in children with
Cerebral Malaria (46.67 \%) and prevalence statistically different from that in the control group $(\mathrm{P}, 0 \cdot 001)^{16}$.

In a case-control study in Southern Ethiopia on 356 (89 malnourished and 267 nourished) under-five children who had previous exposure to Plasmodium infection was found to be a predictor for the manifestation of malnutrition in under-five children $(\mathrm{P}=0.02[\mathrm{OR}=1.87, \mathrm{CI}=1.115-3.138])^{17}$.

Accordingly, a cross- sectional survey was conducted during the peak malaria season in November 2014, in Pitoa and Mayo-Oulo Health Districts. Three hundred and sixty-eight children aged 6months-5 years were enrolled and found that there was a significant association between malaria and malnutrition [OR $=1.89$, (95\% CI: $1.12-3.19)$; $(\mathrm{p}=0.017)]$. Malnutrition was also strongly associated with malaria status $[\mathrm{OR}=2.07,(95 \% \mathrm{CI}$ : 1.22-3.53); $(\mathrm{p}=0.007)]^{18}$.

A study in Zambian children published in 2000 has been found an effect of malaria infection on growth. Children with malaria parasitemia and elevated serum concentrations of C-reactive protein (CRP) at baseline showed significantly lower growth $(0.76$ $0.4 \mathrm{~cm} ; \mathrm{n}=33$ ) compared with children without evidence of malaria infection after 3 months of follow-up (1.0 $60.6 \mathrm{~cm} ; \mathrm{n}=17)(\mathrm{P}=0.03)^{19}$.

A link between malaria and decreased linear growth was also seen in 442 children aged 0-72 months in Peru, which was published in 2012. Each episode of Plasmodium vivax infection predicted a $0.070-\mathrm{cm} /$ episode decrease in linear velocity over 4 months and a $0.083-\mathrm{cm} /$ episode decrease over 6 mo. Similar to several of the studies in this review, children under observation were actively diagnosed and treated for malaria, which creates the potential for attenuation of the effect of malaria on stunting ${ }^{20}$.

A study published in 1977 in 214 Gambian children produced an effect estimate of the association of malaria with growth faltering of $27 \mathrm{~mm} /$ month (SE $=6.0 \mathrm{~mm}$ ), but the effect was not significant ${ }^{21}$.

An observational study that took place in Senegal in 874 children aged 3-6 y found no association between malaria and stunting. Stunted children were disproportionately lost to follow-up due to 
hospitalization for chronic malnutrition, which may have reduced their ability to find an effect ${ }^{22}$.

A recent study in Brazil found that $\$ 1$ episode of malaria was associated with elevated odds of inadequate growth velocity (adjusted OR: 4.0; 95\% CI: $1.4,11.4 ; \mathrm{P}=0.008$ ). However, these effects were limited to children between the ages of 5 and $10 \mathrm{y}$. There was no effect seen in children $<5 \mathrm{y}$ of age, so the effect found in this study is not relevant to the LiST model, which includes only children $<5$ y old ${ }^{23}$.

Table 01: Prevalence of clinical and asymptomatic malaria parasitaemia as affected by nutritional status $^{15}$

\begin{tabular}{|l|c|c|c|c|}
\hline \multirow{2}{*}{ Parameter } & Status & $\mathrm{N}$ & $\begin{array}{c}\text { Clinical } \\
\text { malaria } \\
\text { parastaemia } \\
\text { prevelence }\end{array}$ & $\begin{array}{c}\text { Asymptomatic } \\
\text { malaria } \\
\text { parastaemia } \\
\text { prevelence }\end{array}$ \\
\hline Malnourished & Yes & 103 & 8.88 & 0.68 \\
\cline { 2 - 5 } & No & 348 & 0.003 & 0.41 \\
\hline Stunted & Yes & 77 & 6.59 & 0.01 \\
\cline { 2 - 5 } & No & 374 & 0.01 & 0.91 \\
\hline \multirow{2}{*}{ Underweight } & Yes & 37 & 7.28 & 0.59 \\
\cline { 2 - 5 } & No & 414 & 0.007 & 0.44 \\
\hline Wasted & Yes & 31 & 0.08 & 0.08 \\
\cline { 2 - 5 } & No & 420 & 0.78 & 0.77 \\
\hline
\end{tabular}

\section{Discussions}

Inadequate data from different hospital-based studies regarding mortality from malaria suggest that poor nutritional status at admission is related to an increased risk of death. Different nutrient deficiencies like protein-energy malnutrition, have also been connected to an increased risk of malaria morbidity ${ }^{24}$. There was no evidence of any community-based studies that related nutritional status to mortality among children with slideconfirmed malarial parasitaemia. In one community-based study of nutritional status and allcause mortality conducted in a region of the Democratic Republic of the Congo (formerly Zaire) where malaria is endemic, an association was observed between severe malnutrition and all-cause mortality but not mild or moderate malnutrition ${ }^{25}$. The clinical signs of severe malaria are similar to pneumonia, septicemia, and other infectious diseases $^{26}$ and diagnosing malaria using non- specific indicators, such as fever alone, can be misleading and may mask potential associations with malnutrition.

Whether there is an association between malaria and malnutrition is a controversial issue, in that some studies report that children exposed to malaria have a higher risk of becoming malnourished, while others have found that no association exists. A study found a statistically significant association between malaria and malnutrition $(\mathrm{P}<0.05)$. Under-five children previously exposed to Plasmodium infection were found to be 1.87 times more likely to develop malnutrition than non-exposed children. These findings are similar to those of many other studies, which have reported that malaria can affect the nutritional status of children. A study conducted in the rural community of Amazonian region indicated that children who suffered malaria episodes presented worse anthropometric parameters. Likewise, in a study carried out in a holoendemic malaria area of Tanzania, catch-up growth was seen in those children who used ITNs, which suggests that Plasmodium infection plays a role in the etiology of malnutrition ${ }^{17}$.

Malaria episodes interferes with the nutritional status of children, with notable reduced linear growth velocity as well as impairment in other indices associated with chronic malnutrition, even after adjustment for other factors. Suffering from one or multiple episodes of malaria had a significant negative effect on the linear growth velocity of children, especially amongst children between 5 and 10 months of age. This important finding suggests that even in areas where malaria transmission is low and $P$. vivax is the most prevalent species, the infection may influence the physical development of under-five children ${ }^{23}$.

Thus, at this time, there is insufficient evidence to support a direct causal link from malaria to stunting. That is not to say that it is impossible that such a link exists. A consistent limitation among the studies is that children under observation were actively diagnosed and treated for malaria. Performing this ethical responsibility may have had the unintended consequence of altering the natural 
history of the infection and preventing the possible effect of malaria on stunting. In addition, many of the observational studies and trials included children $>2 \mathrm{y}$ of age, and previous studies have shown that there is less potential to change a childs eventual HAZ status after the age of 2 (34). Furthermore, the Hawthorne effect (i.e., changes in subject's behavior due to their awareness of being under observation) could have affected the results of the studies, and loss to follow-up could have attenuated the effect of malaria on stunting or reduced the power of the studies to be able to detect an effect. Furthermore, the overwhelming majority of studies took place in African countries where Plasmodium falciparum is the dominant circulating malaria species (35). It would be interesting to learn whether the results would be different in regions where Plasmodium vivax infections are more dominant, because $P$. falciparum infections are typically acute, whereas P. vivax infections can form dormant hypnozoites in the liver and cause chronic relapses (36). The HAZ indicator, being an indicator of chronic malnutrition, may be more responsive to the prevention of chronic infection and inflammation. In addition, the chronic nature of the HAZ indicator may present a limitation to the studies as a whole. The duration of the studies may have been insufficient to detect the effect of malaria or malaria prevention and treatment interventions on stunting ${ }^{21}$.

\section{Conclusions}

It is clear that despite the limitations of each study, malnutrition considered as a major burden associated with malaria for under-five children in most of the developing countries. This review also shows that despite three decades of work considerable research still needs to be done. Moreover, the relation between the different components of malnutrition: wasting, stunting, and micronutrient deficiencies need further study so that their relation with malaria in under-five children is better understood. Such information could help in designing intervention programmes that promote the survival of children.

\section{Acknowledgements}

We thank our colleagues who helped to compile the reports we reviewed.

\section{References}

1. The World Health Organization (WHO): Factsheet on the World Malaria Report 2012: access on: 20/05/2020 Available at: https://www.who.int/malaria/media/world_mal aria_report_2012_facts/

2. Hassen H Y, Ali J H: The association between chronic undernutrition and malaria among Ethiopian children aged 6 - 59 months: A facility-based case-control study: S Afr J Child Health 2016;10(1):63-67. DOI:10.7196/SAJCH. 2016.v10i1.1052

3. Rice A.L, Sacco L, Hyder A, Black R.E: Malnutrition as an underlying cause of childhood deaths associated with infectious diseases in developing countries: Bulletin of the World Health Organization, 2000, 78: 12071221.

4. Nubé M, Sonneveld BG. The geographical distribution of underweight children in Africa. Bull World Health Organ. 2005; 83:764-70.

5. Katona P, Katona-Apte J. The interaction between nutrition and infection. Clin Infect Dis. 2008;46:1582-8.

6. Maggini S, Wenzlaff S, Hornig D. Essential role of vitamin $\mathrm{C}$ and zinc in child immunity and health. J Int Med Res. 2010;38:386-414.

7. Scrimshaw NS, SanGiovanni JP. Synergism of nutrition, infection, and immunity: an overview. Am J Clin Nutr. 1997;66(Suppl):464-77.

8. Keusch GT. The history of nutrition: malnutrition, infection and immunity. J Nutr. 2003;133(Suppl):336S-40S.

9. Man WD et al. Nutritional status of children admitted to hospital with different diseases and its relationship to outcome in The Gambia, West Africa. Tropical Medicine and International Health 1998, 3: 678-686.

10. Faye $\mathrm{O}$ et al. [Malaria lethality in Dakar pediatric environment: study of risk factors.] 
Me' decine Tropicale (Marseilles), 1998, 58: 361-364 (in French).

11. Olumese PE et al. Protein energy malnutrition and cerebral malaria in Nigerian children. Journal of Tropical Pediatrics, 1997, 43: 217219.

12. Randriamiharisoa FA et al. [Epidemiological data on children hospitalized with malaria from 1983 to 1992.] Archives de l'Institut Pasteur de Madagascar, 1993, 60: 38-42 (in French).।

13. Razanamparany MS et al. [The malaria epidemic in Antananarivo from 1983 to 1994 as seen through the Pediatric Service A in the Befelatanana General Hospital.] Sante' , 1995, 5: 382-385 (in French).

14. Renaudin P. [Evaluation of the nutritional status of children less than 5 years of age in Moundou, Chad: correlations with morbidity and hospital mortality.] Me' decine Tropicale (Marseilles), 1997, 57: 49-54.

15. Sumbele et al.: Nutritional status of children in a malaria meso endemic area: cross sectional study on prevalence, intensity, predictors, influence on malaria parasitaemia and anaemia: BMC Public Health (2015) 15:1099,

DOI 10.1186/s12889-015-2462-2

16. Mfonkeu J. B. P, Gouado I, Kuate H. F, Zambou O, Combes V, Grau E. R, Zollo P. H. A: Biochemical markers of nutritional status and childhood malaria severity in Cameroon: British Journal of Nutrition (2010), 104, 886892, doi:10.1017/S0007114510001510

17. Gone T, Lemango F, Eliso E, Yohannes S, Yohannes T: The association between malaria and malnutrition among under-five children in Shashogo District, Southern Ethiopia: a casecontrol study, Infectious Diseases of Poverty (2017) 6:9, DOI 10.1186/s40249-016-0221-y

18. Sakwe N, Bigoga J, Ngondi J, Njeambosay B, Esemu L, Kouambeng C, Nyonglema $\mathrm{P}$, Seumen C, Gouado I, Oben J: Relationship between malaria, anaemia, nutritional and socio-economic status amongst under-ten children, in the North Region of Cameroon: A cross-sectional assessment: PLOS ONE, June 21, 2019

19. Hautvast JL, Tolboom JJ, Willems JL, Mwela $\mathrm{CM}$, Monnens L. Consequences of infections for three month length increment in young children in rural Zambia. Acta Paediatr 2000;89:296-301.

20. Lee G, Yori P, Olortegui MP, PanW, Caulfield L, Gilman RH, Sanders JW, Delgado HS, Kosek M. Comparative effects of vivax malaria, fever and diarrhea on child growth. Int $J$ Epidemiol 2012;41:531-9.

21. Rowland MG, Cole TJ, Whitehead RG. A quantitative study into the role of infection in determining nutritional status in Gambian village children. Br J Nutr 1977;37:441-50.

22. Friedman JF, Kwena AM, Mirel LB, Kariuki SK, Terlouw DJ, Phillips-Howard PA, Hawley WA, Nahlen BL, Shi YP, ter Kuile FO. Malaria and nutritional status among pre-school children: results from cross-sectional surveys in western Kenya. Am J Trop Med Hyg 2005;73: 698-704.

23. Alexandre MA, Benzecry SG, Siqueira AM, Vitor-Silva S, Melo GC, Monteiro WM, Leite HP, Lacerda MV, Alecrim Md. The association between nutritional status and malaria in children from a rural community in the Amazonian region: a longitudinal study. PLoS Negl Trop Dis 2015;9:1-15.

24. Shankar AH. Nutritional modulation of malaria morbidity and mortality. The Journal of Infectious Diseases, 2000, 182: S37-S53.

25. Van den Broeck J, Eeckels R, Vuylsteke J. Influence of nutritional status on child mortality in rural Zaire. Lancet, 1993, 341: 1491-1495.

26. Greenwood B. Malaria mortality and morbidity in Africa. Bulletin of the World Health Organization, 1999, 77: 617-618. 